\title{
A robust dissociation among the language, multiple demand, and default mode networks: evidence from inter-region correlations in effect size
}

\author{
Zachary Mineroff ${ }^{\# 1}$, Idan Asher Blank ${ }^{\# 1}$, Kyle Mahowald ${ }^{1}$, and Evelina Fedorenko ${ }^{1,2,3}$ \\ ${ }^{1}$ Massachusetts Institute of Technology \\ ${ }^{2}$ Harvard Medical School \\ ${ }^{3}$ Massachusetts General Hospital \\ \# These authors contributed equally to this work.
}

\begin{abstract}
Complex cognitive processes, including language, rely on multiple mental operations that are carried out by several large-scale functional networks in the frontal, temporal, and parietal association cortices of the human brain. The central division of cognitive labor is between two fronto-parietal bilateral networks: (a) the multiple demand (MD) network, which supports executive processes, such as working memory and cognitive control, and is engaged by diverse task domains, including language, especially when comprehension gets difficult; and (b) the default mode network (DMN), which supports introspective processes, such as mind wandering, and is active when we are not engaged in processing external stimuli. These two networks are strongly dissociated in both their functional profiles and their patterns of activity fluctuations during naturalistic cognition. Here, we focus on the functional relationship between these two networks and a third network: (c) the fronto-temporal left-lateralized "core" language network, which is selectively recruited by linguistic processing. Is the language network distinct and dissociated from both the MD network and the DMN, or is it synchronized and integrated with one or both of them? Recent work has provided evidence for a dissociation between the language network and the MD network. However, the relationship between the language network and the DMN is less clear, with some evidence for coordinated activity patterns and similar response profiles, perhaps due to the role of both in semantic processing. Here we use a novel fMRI approach to examine the relationship among the three networks: we measure the strength of activations in different language, $\mathrm{MD}$, and DMN regions to functional contrasts typically used to identify each network, and then test which regions co-vary in their contrast effect sizes across 60 individuals. We find that effect sizes correlate strongly within each network (e.g., one language region and another language region, or one DMN region and another DMN region), but show little or no correlation for region pairs across networks (e.g., a language region and a DMN region).
\end{abstract}

Corresponding authors: Idan A. Blank (iblank@ mit.edu) or Ev Fedorenko (evelina9@ mit.edu).

Publisher's Disclaimer: This is a PDF file of an unedited manuscript that has been accepted for publication. As a service to our customers we are providing this early version of the manuscript. The manuscript will undergo copyediting, typesetting, and review of the resulting galley proof before it is published in its final citable form. Please note that during the production process errors may be discovered which could affect the content, and all legal disclaimers that apply to the journal pertain. 
Thus, using our novel method, we replicate the language/MD network dissociation discovered previously with other approaches, and also show that the language network is robustly dissociated from the DMN, overall suggesting that these three networks contribute to high-level cognition in different ways and, perhaps, support distinct computations. Inter individual differences in effect sizes therefore do not simply reflect general differences in vascularization or attention, but exhibit sensitivity to the functional architecture of the brain. The strength of activation in each network can thus be probed separately in studies that attempt to link neural variability to behavioral or genetic variability.

\section{Keywords}

functional MRI; language; multiple demand network; default mode network; individual-subject analyses

\section{Introduction}

High-level cognition is supported by the frontal, temporal, and parietal association cortices, which have vastly expanded in the human brain compared to the brains of our closest primate relatives (e.g., Buckner \& Krienen, 2013). These cortices are not organized into individual, "isolated" regions, but rather consist of multiple large-scale "networks": sets of regions that share structural and functional properties (e.g., Fox et al., 2005; Golland et al., 2007; Hagmann et al., 2008; Toro et al., 2008; Seeley et al., 2009; Power et al., 2011; Raznahan et al., 2011; van den Heuvel \& Sporns, 2011; Vértes et al., 2012; Wu et al., 2011; Yeo et al., 2011; Bernard et al., 2012; Chen et al., 2012; de Pasquale et al., 2012; Kalcher et al., 2012; Konopka et al., 2012; Alexander-Bloch et al., 2013; Crossley et al., 2013; Zilles et al., 2015). How many such networks cover the association cortices, what the boundaries of each are, and which aspects of cognition each supports is still debated.

Most agree that the central division of cognitive labor is between two networks: (a) the fronto-parietal bilateral multiple demand (MD) network (e.g., Duncan \& Owen, 2000; Duncan, 2010, 2013; see also Cabeza \& Nyberg, 2000; Braver et al., 2003; Cole \& Schneider, 2007; Dosenbach et al., 2007, among others), which supports diverse goaldirected behaviors (e.g., Fox et al., 2005; Stiers et al., 2010) and is modulated by general cognitive effort (e.g., Duncan \& Owen, 2000; Fedorenko et al., 2013; Hugdahl et al., 2015); and (b) the fronto-parietal bilateral default mode network (DMN; Raichle et al., 2001; Buckner et al., 2008; Andrews-Hanna et al., 2010; Humphreys et al., 2015), which supports more "restful", internally-oriented, processes (e.g., Gusnard \& Raichle, 2001; Raichle et al., 2001) such as mind-wandering, reminiscing about the past, and imagining the future (e.g., Buckner et al., 2008; Spreng et al., 2009). The distinct and complementary functions of these two networks have long been recognized (e.g., Greicius et al., 2003; Fox et al., 2005; Fransson, 2005; Golland et al., 2007; Uddin et al., 2009), and each of them appears to have a homolog in non-human primates (MD: Mitchell et al., 2016; DMN: Mantini et al., 2011).

However, these networks are not the only contributors to complex cognition, at least in humans. In particular, in the human brain, a set of frontal and temporal regions appear to selectively support language processing (e.g., Fedorenko et al., 2011; Monti et al., 2012). 
Here, we probe the relationship between this left-lateralized fronto-temporal "core" language network (e.g., Fedorenko et al., 2010; Fedorenko \& Thompson-Schill, 2014) and the MD and default mode networks. Is the language network distinct and dissociated from the MD network and the DMN, or is it functionally integrated with one or both of them? One might hypothesize that the language network is integrated, to some extent, with the MD network given that language processing requires general attention, working memory, and cognitive control (e.g., Gibson, 1998; Hsu \& Novick, 2016; Nozari \& Novick, 2017; for reviews, see Novick et al., 2010; Fedorenko, 2014). And one might also hypothesize that the language network is integrated with the DMN given that a lot of our introspective processing plausibly draws on verbal resources (e.g., Vygotsky, 1962, 2012; Sokolov, 1972; Zivin, 1979; Carruthers, 2002; Pleh, 2002; Schrauf, 2002; Morin \& Michaud, 2007).

Until recently, researchers have actually not explicitly distinguished between the language and the MD networks, especially in the frontal lobes, where subsets of each network reside side by side within the region known as "Broca's area" (Fedorenko et al., 2012). However, recent work has established that these networks are spatially and functionally distinct based on three converging lines of evidence. First, language and MD regions exhibit distinct functional profiles: whereas MD regions are recruited across many cognitive tasks, language regions respond selectively during language processing and are not engaged by a wide range of non-linguistic processes, including arithmetic, working memory, cognitive control, music perception, and action observation (e.g., Fedorenko et al., 2011; Monti et al., 2012; Pritchett et al., in press; for a review, see Fedorenko \& Varley, 2016). Second, language and MD regions show distinct patterns of fluctuations in neural activity during naturalistic cognition. For example, Blank et al. (2014; replicated in Paunov et al., in revision) compared fluctuations in the fMRI BOLD signal across language and MD regions either during "rest" or while participants listened to stories. In both conditions, the average pairwise correlations among language regions (see also Hampson et al., 2002; Turken and Dronkers, 2011; Yue et al., 2013) and among MD regions (see also Dosenbach et al., 2007; Seeley et al., 2007;

Hampshire et al., 2012) were significantly higher than correlations between pairs of regions straddling the two networks, which were close to zero. This dissociation was further supported by data-driven clustering of regional BOLD signal time-courses, which grouped language and MD regions separately. And third, damage to language vs. MD regions leads to distinct patterns of cognitive deficits (e.g., Woolgar et al., 2018). (Of course, it is important to keep in mind that this dissociation between the language and the MD networks does not imply that the two networks cannot or do not work together in the service of certain complex cognitive tasks. Indeed some complex language processing tasks engage regions of both networks (e.g., Stromswold et al., 1996; Stowe et al., 1998; Constable et al., 2004; Chen et al., 2006; Nieuwland et al., 2007; Novais-Santos et al., 2007; January et al., 2009; Ye \& Zhou, 2009; Peelle et al., 2010; Sebastian et al., 20122; Barde et al., 2012; McMillan et al., 2012, 2013; Wild et al., 2012; Brownsett et al., 2013; de Bruin et al., 2014; Geranmayeh et al., 2014a,b; De Baene et al., 2015; Gauvin et al., 2016; Henderson et al., 2016; Hsu et al., 2017). We return to this point in the discussion).

The relationship between the language network and the DMN remains less clear. For example, algorithms that cluster voxels across the brain based on similarities in their activity fluctuations during rest often produce a cluster whose topography resembles a union of the 
language network and the DMN (e.g., Yeo et al., 2011). However, interpreting the resulting cluster in functional terms can be difficult (for a discussion see e.g., Blank et al., 2014; Blank \& Fedorenko, 2017) and must rely on logically precarious "reverse inference" from anatomical coordinates back to cognitive processes (Poldrack, 2006, 2011). Furthermore, at least some of the language regions appear to deactivate during some demanding cognitive tasks (e.g., Fedorenko et al., 2011; see also Figure 1, top panel), which is one functional signature of the DMN—although, unlike DMN regions, language regions increase their activity during difficult language processing tasks (e.g., Blank et al., 2016). Finally, both language and DMN regions have been linked to semantic / conceptual processing (e.g., Binder et al., 2009; Wirth et al., 2011; Jackson et al., 2016; Mattheis et al., 2018). However, damage to each network produces distinct behavioral deficits: deficits in language comprehension and production for the language network (e.g., Bates et al., 2003; Ojemann et al., 2003; Mesulam et al., 2015; Mirman et al., 2015;), and deficits in e.g., autobiographical memory retrieval for the DMN (e.g., Damasio \& Van Hoesen, 1983; Philippi et al., 2015).

To shed further light on the relationship among the language, multiple demand, and default mode networks, here we characterize and directly compare their functional properties using fMRI. We first examine the basic response profiles of language, MD, and DMN regionsdefined functionally in each of 60 individual participants-and show that the profiles are clearly distinct. We then use a novel approach to probe the relationship among the three networks, testing whether the strength of functional responses in these three networks covaries across participants. This approach is inspired by several recent findings. First, different language regions robustly co-vary across individuals in their respective effect sizes for a contrast between reading sentences and reading lists of nonwords (Mahowald \& Fedorenko, 2016). Second, different MD regions co-vary across individuals in their respective effect sizes for a contrast between hard and easy spatial working memory task (Assem et al., in revision). Importantly, these effect sizes appear to be highly stable within participants across runs and scanning sessions, suggesting that they tap some time-invariant idiosyncratic properties of individual brains. Here, we extend this study of effect-size correlations from pairs of regions within a single network to pairs of regions across different networks. If these effect size measures reflect some highly general properties, like the degree of brain vascularization or fluid intelligence levels, then all three networks should co-vary in these measures across individuals. However, if these measures are sensitive tofunctional dissociations among distinct brain networks, we expect the language and MD networks to show little co-variation in these measures across participants, consistent with prior studies (e.g., Fedorenko et al., 2011; Fedorenko et al., 2012; Blank et al., 2014; Blank \& Fedorenko, 2017; Paunov et al., in revision). Critically, if effect size measures indeed respect such functional distinctions, then the degree to which the language and DMN regions co-vary across individuals could indicate the extent of functional association between these two networks. Toforeshadow our conclusions, these measures replicate the robust language-MD dissociation, and show that the language network and the DMN are also robustly dissociated. 


\section{Methods}

\section{Participants}

Sixty participants (41 females) between the ages of 19 and 45-students at MIT and members of the surrounding community-were paid for their participation. Participants were right-handed native speakers of English, naïve to the purposes of the study. All participants gave informed consent in accordance with the requirements of MIT's Committee On the Use of Humans as Experimental Subjects (COUHES).

\section{Design, materials, and procedure}

Each participant performed two tasks that were designed to localize the functional networks of interest: a reading task for the language network (adapted from Fedorenko et al., 2010) and a spatial working memory (WM) task for the MD network and DMN (from Fedorenko et al., 2011). Some participants also completed one or two additional tasks for unrelated studies. The scanning session lasted approximately 2 hours.

\section{Language localizer task}

Participants read sentences (e.g., NOBODY COULD HAVE PREDICTED THE EARTHQUAKE IN THIS PART OF THE COUNTRY) and lists of unconnected, pronounceable nonwords (e.g., U BIZBY ACWORRILY MIDARAL MAPE LAS POME U TRINT WEPS WIBRON PUZ) in a blocked design. Each stimulus consisted of twelve words/nonwords. For details of how the language materials were constructed, see Fedorenko et al. (2010). The materials are available at http://web.mit.edu/evelina9/www/funcloc/ funcloc_localizers.html. The sentences > nonword-lists contrast has been previously shown to reliably activate high-level language processing regions and to be robust to the materials, task, and modality of presentation (Fedorenko et al., 2010; Fedorenko et al., 2011;

Mahowald \& Fedorenko, 2016; Scott et al., 2016). The brain regions activated by this contrast have been shown to be sensitive to both word-level meanings and combinatorial syntactic and semantic processing (e.g., Fedorenko et al., 2010, 2012; Menenti et al., 2010; Blank et al., 2016).

Stimuli were presented in the center of the screen, one word/nonword at a time, at the rate of $450 \mathrm{~ms}$ per word/nonword. Each stimulus was preceded by a $100 \mathrm{~ms}$ blank screen and followed by a 400ms screen showing a picture of a finger pressing a button, and a blank screen for another $100 \mathrm{~ms}$, for a total trial duration of $6 \mathrm{~s}$. Participants were asked to press a button whenever they saw the picture of a finger pressing a button. This task was included to help participants stay alert and awake.

Condition order was counterbalanced across runs. Experimental blocks lasted 18s (with 3 trials per block), and fixation blocks lasted 14s. Each run (consisting of 5 fixation blocks and 16 experimental blocks) lasted 358s. Each participant completed 2 runs.

\section{Spatial working memory task}

Participants had to keep track of four (easy condition) or eight (hard condition) sequentially presented locations in a $3 \times 4$ grid (Fedorenko et al., 2011). In both conditions, participants 
performed a two-alternative forced-choice task at the end of each trial to indicate the set of locations they just saw. The hard > easy contrast has been previously shown to robustly activate MD regions (Fedorenko et al., 2013; Blank et al., 2014), which also have been shown to respond to difficulty manipulations across many diverse tasks (e.g., Duncan \& Owen, 2000; Fedorenko et al., 2013; Hugdahl et al., 2015). The reverse contrast, easy > hard, robustly activates DMN regions, in line with prior work using similar tasks and contrasts (McKiernan et al., 2003; Park et al., 2010; Leech et al., 2011).

Stimuli were presented in the center of the screen across four steps. Each of these steps lasted for $1000 \mathrm{~ms}$ and presented one location on the grid in the easy condition, and two locations in the hard condition. Each stimulus was followed by a choice-selection step, which showed two grids side by side. One grid contained the locations shown on the previous four steps, while the other contained an incorrect set of locations. Participants were asked to press one of two buttons to choose the grid that showed the correct locations.

Condition order was counterbalanced across runs and participants. Experimental blocks lasted 32s (with 4 trials per block), and fixation blocks lasted 16s. Each run (consisting of 4 fixation blocks and 12 experimental blocks) lasted 448s. Each participant completed 2 runs.

\section{fMRI data acquisition}

Structural and functional data were collected on the whole-body, 3 Tesla, Siemens Trio scanner with a 32-channel head coil, at the Athinoula A. Martinos Imaging Center at the McGovern Institute for Brain Research at MIT. T1-weighted structural images were collected in 176 sagittal slices with $1 \mathrm{~mm}$ isotropic voxels $(\mathrm{TR}=2530 \mathrm{~ms}, \mathrm{TE}=3.48 \mathrm{~ms})$. Functional, blood oxygenation level dependent (BOLD), data were acquired using an EPI sequence (with a 90oflip angle and using GRAPPA with an acceleration factor of 2), with the following acquisition parameters: thirty-one $4 \mathrm{~mm}$ thick near-axial slices acquired in the interleaved order (with $10 \%$ distance factor), $2.1 \mathrm{~mm} \times 2.1 \mathrm{~mm}$ in-plane resolution, $\mathrm{FoV}$ in the phase encoding $(A>>P)$ direction $200 \mathrm{~mm}$ and matrix size $96 \mathrm{~mm} \times 96 \mathrm{~mm}, \mathrm{TR}=2000 \mathrm{~ms}$ and $\mathrm{TE}=30 \mathrm{~ms}$. The first $10 \mathrm{~s}$ of each run were excluded to allow for steady state magnetization.

\section{fMRI data preprocessing}

MRI data were analyzed using SPM5 and custom Matlab scripts (available in the form of an SPM toolbox from http://www.nitrc.org/projects/spm_ss). Each participant's data were motion corrected and then normalized into a common brain space (the Montreal Neurological Institute (MNI) template) and resampled into $2 \mathrm{~mm}$ isotropic voxels. The data were then smoothed with a 4mm FWHM Gaussian filter and high-pass filtered (at 200s). Effects were estimated using a General Linear Model (GLM) in which each experimental condition was modeled with a boxcar function (modeling entire blocks) convolved with the canonical hemodynamic response function (HRF).

\section{Defining individual functional regions of interest (fROls)}

All the analyses described below were performed on the responses in regions of interest that were defined functionally in each individual participant (e.g., Saxe et al., 2006; Fedorenko et 
al., 2010; Nieto-Castañón \& Fedorenko, 2012). Three sets of functional regions of interest (fROIs) were defined, one set for each of the three networks. To do so, we used the Groupconstrained Subject-Specific (GSS) approach developed in Fedorenko et al. (2010; Julian et al., 2012). In particular, fROIs were constrained tofall within a set of "masks" which marked the expected gross locations of activations for the relevant contrast. For the language network, the masks were generated based on a group-level representation of data from 220 participants (see Figure 1; these masks are similar to the masks originally reported in Fedorenko et al., 2010 based on 25 participants, except that the left anterior temporal and left mid-anterior temporal masks are grouped together, and the left mid-posterior temporal and left posterior temporal masks are grouped together). In addition, given that both the MD network and the DMN are bilateral, we defined the RH homologs of the LH language regions by transposing the LH masks onto the RH, as in Blank et al. (2014). fROIs were then defined in each RH mask, and were allowed the differ from their left homologues in their precise locations within these masks. For the MD and DMN networks, the masks were mostly anatomical regions from the Tzourio-Mazoyer et al. (2002) atlas, selected based on the prior literature (see Figure 1; the anatomical MD masks were the same as those used in Fedorenko et al., 2013 and Blank et al., 2014). The only exceptions are the bilateral Temporo-Parietal Junction masks for the DMN, which were created based on a randomeffects analysis for a functional contrast from a Theory of Mind localizer (false belief $>$ false photograph) from 462 participants (Dufour et al., 2013).

For each participant, each set of masks was intersected with their individual activation map for the relevant contrast (i.e., sentences > nonwords for the language network, hard > easy spatial WM for the MD network, and easy > hard spatial WM for the DMN). Within each mask, the voxels were then sorted based on their $t$-values for the relevant contrast, and the top $10 \%$ of voxels were selected as that participant's fROI. This top $n \%$ approach ensures that the fROIs can be defined in every participant- thus enabling us to generalize the results to the entire population (Nieto-Castañón \& Fedorenko, 2012) — and that fROI sizes are the same across participants.

For the language network, twelve fROIs were defined in each participant, six in the left (L) hemisphere and six in the right (R) hemisphere. These included six fROIs on the lateral surface of the frontal cortex in the inferior frontal gyrus (L/R IFG) and its orbital part (L/R IFGorb) as well as in the middle frontal gyrus (L/R MFG); and six fROIs on the lateral surface of the temporal and parietal cortex, in the anterior temporal cortex (L/R AntTemp), posterior temporal cortex (L/R PostTemp), and angular gyrus (L/R AngG).

For the MD network, eighteen fROIs were defined in each participant, nine in each hemisphere. These included the opercular part of the inferior frontal gyrus (L/R IFGop), the middle frontal gyrus (L/R MFG) and its orbital part (L/R MFGorb), the precentral gyrus (L/R PrecG), the insular cortex (L/R Insula), the supplementary motor area (L/R SMA), the inferior parietal cortex (L/R InfPar), the superior parietal cortex (L/R SupPar), and the anterior cingulate cortex (L/R AntCing).

Finally, for the DMN, ten fROIs were defined in each participant, five in each hemisphere. These included the posterior cingulate cortex (L/R PostCing), four medial frontal regions 
(L/R FrontMedOrb and L/R FrontMedSup), the precuneus (L/R Precuneus), and the temporoparietal junction (L/R TPJ).

\section{Examining the functional response profiles of fROls}

To estimate the responses of the fROIs to the conditions used to define them, we used an across-runs cross-validation procedure. In particular, for each relevant contrast (sentences > nonwords for the language regions, hard > easy for the MD regions, and easy > hard for the DMN regions), fROIs were defined for each participant based on data from only the first run, and their responses were then estimated using data from the second run. This procedure was then repeated using the second run to define the fROIs and the first run to estimate the responses. Finally, the responses were averaged across these two iterations to derive a single response magnitude for each condition in a given fROI/participant. This cross-validation procedure allows one to use all of the data for defining the fROIs as well as for estimating their responses (for discussion, see Nieto-Castanon \& Fedorenko, 2012), while ensuring the independence of the data used for fROI definition and for response estimation (e.g., Kriegeskorte et al., 2009).

In order to compare the functional profiles across the three networks, we also estimated the responses of the fROIs to conditions that were not used to define them (i.e., hard and easy WM for the language network, sentences and nonwords for the MD network and DMN). Here, we used all of the data from the localizer task (i.e., both runs) to define the fROIs, and all of the data from the other task to estimate their responses.

Second-level analyses (repeated measures $t$-tests) were performed on these extracted response magnitude values, using false discovery rate (FDR) correction (Benjamini \& Yekutieli, 2001) for the number of fROIs in each network. Pairwise comparisons of localizer effects across networks were tested using linear, mixed-effects regression models implemented with the "Imer" toolbox in R (Bates et al., 2015). These models included a fixed effect for network, a random slope of network by participant, and a random intercept by fROI. The fixed effect estimates were contrasted to each other using the "multcomp" package. Because these analyses were carried out to replicate previous findings, hypotheses were one-tailed.

\section{Examining inter-individual co-variation in effect sizes of the three networks}

Descriptive statistics.-For each functional contrast (sentences > nonwords, hard > easy WM, easy > hard WM) we computed, across participants, the Pearson correlations in effect size for every pair of fROIs (40 fROIs in total: 12 language fROIs, 18 MD fROIs, and 10 DMN fROIs) (the patterns of results reported below also obtained when using the nonparametric Kendall $\tau$ correlation, and are thus robust to the choice of a particular correlation measure). Then, the correlation values within each network and hemisphere (e.g., all pairwise correlations for regions of the left hemispheric language network) were Fishertransformed and averaged together (this transformation reduces the bias in averaging correlations; see Silver \& Dunlap, 1987). Correlations of fROIs with themselves, which were always equal to 1 , were excluded from this step. Thus, we obtained: 3 networks $\times 2$ hemispheres $=6$ average correlations. Following a similar procedure, we computed the 
average inter-hemispheric correlation for each of the three networks (e.g., the average of all pairwise correlations between one left-hemisphere language region and one righthemisphere language region). Similarly, we also computed the average correlation across pairs of networks, within each hemisphere (e.g., the average of all pairwise correlations between one left-hemisphere language region and one left-hemisphere MD region). Here, we obtained: 3 network pairs $\times 2$ hemispheres $=6$ average correlations. In total, the number of average correlations (within-network, within-hemisphere; within-network, acrosshemispheres; and across-networks, within-hemispheres) was therefore $6+3+6=15$.

For each of these 15 correlations we computed a $95 \%$ confidence interval via a bootstrapping procedure: first, we randomly sampled $5 \times 10^{4}$ sets of $n=60$ participants, with replacement, from the observed data. Then, for each set, we re-computed the 15 correlations as outlined above, yielding - across all sets- 15 distributions of bootstrapped correlations. Finally, for each distribution, we found the interval that contained the middle $95 \%$ of values.

Significance tests.-To test whether fROIs within a certain network $A$ were more strongly correlated among themselves than with fROIs of another network B, we used a permutation approach. Specifically, we randomly shuffled effect sizes across participants for each fROI in A. Under this shuffling, the expected correlations within network $A$ as well as between networks $A$ and $B$ are practically 0 . Consequently, the difference between the mean pairwise correlation within $A$ and the mean pairwise correlation between $A$ and $B$ is also expected to be 0 . The effect of this shuffling procedure therefore corresponds to the null hypothesis that within-network correlations are no different from between-network correlations. Thus, to generate an empirical null distribution, shuffling is repeated many times (here, $5 \times 10-4$ ) and, for each repetition, the mean correlation between $A$ and $B$ is subtracted from the mean correlation within $A$. The resulting distribution is approximately normal, because of both the Fisher-transformation on pairwise correlations and the averaging of these correlations within / across networks. To test the probability that the observed data would be sampled under the null hypothesis, we fit a Gaussian to the null distribution and used its mean and standard deviation to $z$-score the observed data.

We used the same permutation approach to test the laterality of correlations in effect size between fROIs. Namely, we address three questions: (i) whether, for each network, correlations in effect size within each hemisphere are stronger than those across hemispheres; (ii) whether the size of this laterality effect differs across networks; and (iii) whether across-network correlations are also lateralized, such that fROIs in one network are differentially correlated with LH vs. RH fROIs in another network. The results of all of our tests are FDR-corrected for multiple comparisons (Benjamini \& Yekutieli, 2001).

Hierarchical clustering.-The permutation approach described above was used to test hypotheses-driven predictions regarding a tri-partite dissociation among the language, MD and default-mode networks. To use a more data-driven approach for examining our results, we searched for a partition of the 40 fROIs based solely on their pairwise correlations in effect size, ignoring their a-priori network labels. To this end, we first used a hierarchical clustering algorithm (Hartigan, 1975) to gradually connect all fROIs into a binary tree structure: this algorithm starts by joining the most correlated pair of fROIs into a node, and 
proceeds to join other pairs of fROIs and/or higher tree nodes in decreasing order of correlation (correlations between tree nodes are defined as the average pairwise correlation between their respective, constituent fROIs). Following the method of Blank et al. (2014), we then used a modularity-optimization approach tofind the level at which the tree can be "ideally" partitioned into separate branches, each representing a group of fROIs; here, the "ideal" partition is one that maximizes within-branch correlations and minimizes acrossbranch correlations (a measure of "modularity"; see Newman and Girvan, 2004; Gómez et al., 2009).

\section{Results}

\section{Behavioral data}

Behavioral performance on the spatial working memory task was as expected: participants were more accurate and faster on the easy trials (accuracy $=92.65 \pm 1.47 \%$; reaction time (RT) $=1.19 \pm 0.22 \mathrm{~s}$ ) than the hard trials (accuracy $=79.81 \pm 2.39 \%, t_{(59)}=-11.50, p<10^{-16}$, Cohen's $\left.d=1.48 ; \mathrm{RT}=1.47 \pm 0.27 \mathrm{~s}, t_{(59)}=14.19, p<10^{-20}, d=1.83\right)$.

\section{Functional response profiles of the fROls in the three networks}

Replicating previous work, we find robust responses for all localizer contrasts using acrossruns cross-validation (Figure 1). In the language network, the sentences $>$ nonwords effect was highly reliable in each of the LH fROIs $(t s>10.7, p s<0.0001, d s>1.38)$ and in each of the RH fROIs $(t s>4.1, p s<0.0001, d s>0.53)$. In the MD network, the hard $>$ easy effect was highly reliable in each fROI $(t s>10.1, p s<0.0001, d s>1.30)$. And in the DMN network, the easy $>$ hard effect was highly reliable in each fROI ( $t s>7.1, p s<0.0001, d s>0.92)$. Next, we examined the relationship between the language network and each of the other two networks:

Language vs. MD.-Replicating prior work (Fedorenko, et al., 2011), we find no response to the spatial WM task in the language fROIs. None of the fROIs, except for the LMFG fROI, respond above baseline to either the hard spatial WM condition or the easy spatial WM condition (for both conditions, all $t s<1$, n.s., $d<0.13$ ). The LMFG fROI shows above baseline responses to both conditions, in line with what was reported in Fedorenko et al. (2011), and shows a slightly stronger response to the hard than the easy condition, which is not significant after an FDR correction for multiple comparisons $\left(t_{(59)}=1.84\right.$, n.s., $\left.d=0.24\right)$. In both hemispheres, the MD fROIs show an overall higher value for the hard > easy contrast than the language fROIs do (across-network contrast in LH: $0.74, z=7.8, p<10^{-13}$; RH: $0.66, z=5.47, p<10^{-7}$ ).

Replicating Fedorenko et al. (2013), we find that the MD fROIs respond to the language localizer conditions in a manner opposite to the language fROIs. In particular, they respond more to the meaningless and unstructured nonword lists than to the sentence condition $(t s>2.1, p s<0.02, d s>0.27)$. (Interestingly, this pattern holds even though the task in the current version of the language localizer is passive reading (cf. a memory probe task in Fedorenko et al., 2013; see alsofedorenko, 2014).) 
Language vs. DMN.-In line with much prior work (e.g., Gusnard \& Raichle, 2001;

Raichle et al., 2001), we find that the DMN fROIs de-activate to the spatial WM task, with both the hard and the easy condition eliciting a response reliably below the fixation baseline (hard: $t s>7.1, p s<10^{-9}, d s>0.92$; easy: $t s>4.2, p s<10^{-4}, d s>0.54$ ). Critically, in sharp contrast with the language fROIs, none of the DMN fROIs, except for the LTPJ fROI, respond above baseline to sentence comprehension (sentence condition: $t s<1.1$, n.s., $d s<0.14$ ). The LTPJ fROI responds above baseline to the sentence condition $\left(t_{(59)}=4.56, p<10^{-4}, d=0.59\right)$ and reliably more to sentences than nonwords $\left(t_{(59)}=5.14, p<10^{-5}, d=0.66\right)$. Directly comparing the overall response to the sentence condition across the language network and DMN showed that the responses in the former are significantly higher (across-network contrast in LH: $1.31, z=7.99, p<10^{-14}$; RH: $0.67, z=5.08, p<10^{-6}$ ). Therefore, although several language fROIs show a hint of the DMN signature (deactivation to the demanding executive function task, with stronger deactivation to the harder condition, replicating Fedorenko et al., 2011), the functional response in (most of) the DMN fROIs is clearly distinct from that in the language fROIs: language, but not DMN, fROIs respond robustly during language comprehension.

Nevertheless, the fact that the functional profile of the DMN fROI in the LTPJ is similar to that of the language fROI in the LAngG suggests that the two networks may overlap slightly near the junction of left temporal and parietal lobes (see also Deen et al., 2015). Evidence is accumulating that the AngG fROI functionally differs from the rest of the language network based on both functional correlation data (Blank et al., 2014; Chai et al., 2016) and differential responses to linguistic (e.g., Blank et al., 2016) and non-linguistic (e.g., Amit et al., 2017; Pritchett et al., in press) manipulations. Still, we include both the left and right AngG language fROIs in the analyses below because it is conservative with respect to the hypothesis that the three networks are functionally dissociated.

\section{Within- vs. between-network correlations in effect size}

The correlations of effect sizes for all pairs of fROIs are shown in Figure 2. Even before performing any statistical analysis, the dissociation among the language, MD, and default mode networks is visually apparent; the correlations are much higher for pairs of regions within each network than for pairs of regions across networks. A quantitative summary of the effect-size correlations is shown in Figure 3.

In line with previous work, we find a dissociation between the language and MD networks. In the left hemisphere, the average correlation between the sentences > nonwords effect size in one language region and the sentences $>$ nonwords effect size in another language region is $0.54(95 \%$ confidence interval $(\mathrm{CI})=[0.39,0.67])$, similar to what was reported in Mahowald \& Fedorenko (2016). In other words, individuals who show a bigger (smaller) sentences $>$ nonwords effect in a given language region also tend to show bigger (smaller) effects in other language regions. Similarly, the average correlation between the hard > easy effect size in one MD region and the hard > easy effect size in another MD region is 0.66 $\left(\mathrm{CI}_{95 \%}=[0.55,0.76]\right)$, similar to what was reported in Assem et al. (in revision). However, the average correlation between the sentence $>$ nonwords effect size in a language region and the hard > easy effect size in a MD region is $0.18\left(\mathrm{CI}_{95 \%}=[0.03,0.31]\right)$, significantly 
lower than both the within-language $\left(p<10^{-13}\right)$ and within-MD $(\mathrm{p} \approx 0)$ correlations. In other words, individual differences in the size of the sentences $>$ nonwords effect in the language network and the size of the hard > easy effect in the MD network are less predictive of one another, relative to individual differences among fROIs within each network.

Critically, in line with the distinct functional profiles reported above, we also observe a clear dissociation between the language network and the DMN. The average correlation between the sentences $>$ nonwords effect size in a language region and the easy $>$ hard effect size in a DMN region is $0.09\left(\mathrm{CI}_{95 \%}=[-0.08,0.26]\right)$. This is significantly lower than the withinlanguage correlations $(p \approx 0)$ and the within-DMN correlations $\left(0.66, \mathrm{CI}^{95 \%}=[0.58,0.74]\right.$; $p \approx 0$ ). The $\mathrm{DMN}$ is also dissociated from the MD network, with between-network correlations in effect size $\left(0.26, \mathrm{CI}_{95 \%}=[0.08,0.43]\right)$ significantly lower than those within each network (both $p \approx 0$ ).

A similar dissociation among the three networks obtains in the right hemisphere, as shown in Figures 2 and 3: the mean pairwise correlations within the language network (0.47, $\left.\mathrm{CI}_{95 \%}=[0.36,0.58]\right)$ and within the MD network $\left(0.65, \mathrm{CI}_{95 \%}=[0.54,0.75]\right)$ are stronger than the mean language-MD pairwise correlation $\left(-0.05, \mathrm{CI}_{95 \%}=[-0.18,0.11]\right.$; both $\left.p \approx 0\right)$; the correlations within the language network and within the $\mathrm{DMN}\left(0.64, \mathrm{CI}_{95 \%}=[0.54\right.$, 0.74]) are stronger than language-DMN correlations $\left(0.04, \mathrm{CI}_{95 \%}=[-0.10,0.17]\right.$; both $\mathrm{p} \approx$ 0 ); and the correlations within the MD network and within the DMN are stronger than MDDMN correlations $\left(0.25, \mathrm{CI}_{95 \%}=[0.09,0.40] ; p \approx 0\right.$ and $p<10^{-13}$, respectively).

Furthermore, the language network shows a robust lateralization effect in this novel measure, such that LH language fROIs are more correlated among themselves than they are with RH language fROIs (mean inter-hemispheric correlation: $0.22, \mathrm{CI}_{95 \%}=[0.03,0.40]$; $\left.p<10^{-12}\right)$, and the same is true for correlations among RH language fROIs $\left(p<10^{-6}\right)$. These findings are in line with prior functional correlation studies (e.g., Gotts et al., 2013; Blank et al., 2014) and dynamic network modeling studies (e.g., Chai et al., 2016). In contrast, this laterality effect is not observed in either the MD network (mean inter-hemispheric pairwise correlation: $0.62, \mathrm{CI}_{95 \%}=[0.50,0.73]$; compared to LH correlations, $p=0.40$; compared to RH correlations, $p=0.97$ ) or the DMN (mean inter-hemispheric correlation: 0.68, $\mathrm{CI}_{95 \%}=[0.58,0.76]$; compared to LH correlations, $p=1$; compared to RH correlations, $p=0.72$ ). A direct comparison of laterality effects across networks further confirms that they are stronger in the language network than in either the MD or the DMN, for both the $\mathrm{LH}$ (both $p<10^{-5}$ ) and the RH (MD: $p=0.001$; DMN: $p<10^{-5}$ ). A related, potentially interesting observation is that both LH and RH MD fROIs are more correlated with the LH language fROIs than they are with the RH homologues (LH MD fROIs: $p=0.01$; RH MD fROIs: $p=0.003)$. In all other cases, across-network correlations in effect-size do not show such laterality.

These findings, based on hypothesis-driven tests in which sets of fROIs are compared to each other based on a pre-determined division into functional networks, are also supported by a hypothesis-neutral, data-driven analysis (Figure 4). Namely, hierarchically clustering all fROIs into a tree structure based on their pairwise correlations in effect size-without apriori information on their network assignments—recovered the dissociation among 
language, MD and DMN fROIs as well as the associated laterality patterns. Specifically, the tree obtained from this clustering contained four branches precisely corresponding to the LH language, $\mathrm{RH}$ language, bilateral MD, and bilateral DMN networks. This partition had the highest modularity value compared to all other partitions, both finer and grosser, that were licensed by the tree-indicating that it was the overarching organizing principle in our data. In contrast to the branching of language fROIs by hemisphere, the MD and DMN branches were overall organized by inter-hemispheric homology such that many fROIs clustered with their respective contra-lateral homologues before forming larger clusters with one another. Furthermore and interestingly, in spite of the functional similarity between the LTPJ DMN fROI and the LAngG language fROI, as discussed above, they got assigned to their respective networks suggesting that at least in this measure the LTPJ DMN fROI is more similar to other DMN fROIs, and the LAngG language fROI is more similar to other language fROIs.

The results reported above are all based on correlations between effect sizes from the language localizer task (in language fROIs) and effect sizes from the spatial workingmemory task (in MD and DMN fROIs). Differences between these two tasks might therefore trivially account for the functional dissociation among the three networks (or at least between the language network and each of the other two networks). In order to reject this account, we recomputed our critical measure-i.e., inter-region correlations in effect size across participants - based on data from a single localizer contrast across all fROIs. Specifically, we measured the sentences > nonwords effect size in each language, MD and DMN fROI and, then, compared the average inter-regional correlation within the language network to the average language-MD correlation and the average language-DMN correlation. Similarly, we measured the hard > easy (or easy > hard) effect size in each fROI and, then, compared the average interregional correlation within the MD (DMN) network to the corresponding inter-network correlations. The results of this analysis are presented in Table 1. Consistent with our main analysis, they indicate a clear tri-partite dissociation across the three networks.

\section{Discussion}

The current study examined the relationship among three large-scale functional networks that support high-level cognitive processes: the language network, the multiple demand (MD) network, and the default mode network (DMN). To do so, we (a) characterized the functional response profiles of each network, and (b) employed a novel analytic approach that tested, across participants, the correlations in response magnitude among the fROIs within each network vs. between networks. Using both analyses, we replicate the dissociation between the language and MD networks (e.g., Fedorenko et al., 2011; Fedorenko et al., 2013; Blank et al., 2014; Paunov et al., in revision), as well as the wellestablished dissociation between the MD network and the DMN. Critically, we further demonstrate that the language network is also robustly dissociable from the DMN: the former, but not the latter responds strongly during language processing and, whereas regional effect sizes strongly co-vary across individuals within each network, the correlation between the two networks is much weaker. In other words, if an individual shows a strong response to language processing (the functional signature of the language system) in one 
language region, they are also likely to show a strong response in other language regions. Similarly, if an individual shows strong deactivation to a demanding task (the functional signature of the DMN) in one DMN region, they are also likely to show strong deactivation in other DMN regions. However, the strength of the response to language processing in a language region bears much less information about how much a DMN region will deactivate (or how much a region of the MD network will respond) to a demanding task.

These results have three implications. The first one is methodological: inter-individual differences in effect sizes do not simply reflect variability in overall brain properties (e.g., vascularization affecting the fMRI BOLD signal) or in behavioral/cognitive states (e.g., attention). If this were the case, we would expect effect sizes in different brain regions to strongly co-vary across individuals, regardless of their functional profiles. Instead, such inter-individual differences appear to be sensitive to the functional architecture of the brain, respecting its division into distinct, large-scale neural networks. Thus, inter-region correlation in effect size across individuals is a powerful new measure for discovering functional dissociations among neural systems and, possibly, even at a finer grain within each system.

The second implication is theoretical. The prior literature has left the relationship between the language network and the DMN ambiguous. In particular, methods that cluster voxels across the brain based on their respective activity time-courses sometimes recover a network that looks like a combination of the language network and the DMN (e.g., Yeo et al., 2011); this result appears to depend, in part, on the pre-specified number of clusters that such analyses are constrained to produce. Further, although the language regions show no response to non-linguistic demanding tasks (and are thus clearly dissociable from the domain-general MD network), they sometimes show deactivation to such tasks, much like the DMN (e.g., Fedorenko et al., 2011; see also Figure 1, top panel). Finally, both the language and the DMN regions have been linked to semantic / conceptual processing (e.g., Binder et al., 2009; Wirth et al., 2011;Jackson et al., 2016; Mattheis et al., 2018). However, we find a clear and robust functional dissociation between the language network and the DMN. This finding suggests that, in spite of some functional similarities between these two networks, and in spite of the fact that some of their regions lie in close proximity to one another, these two networks are functionally dissociable.

The third implication has to do with an important goal of cognitive neuroscience, to understand how inter-individual variability in brain structure and function relates to behavior and cognition in both healthy individuals and individuals with developmental disorders or mental illness. Although the current study does not inform these questions directly, it makes an important contribution: the dissociation we observed among the three networks suggests that the response strength in each network can be used as a specific neural marker, to be related to behavioral (or genetic) variability (e.g., Dubois \& Adolphs, 2016; Mahowald \& Fedorenko, 2016; Seghier \& Price, 2018; Assem et al., in revision).

Finally, two points are worth clarifying with respect to the functional dissociations among the three networks examined here (or any other brain regions / networks, for that matter). First, simply because two regions or networks are functionally distinct (in whatever 
measure) does not imply that these regions / networks cannot "collaborate" with one another in the service of some task that requires both processes. For example, recognizing an individual presumably engages a number of distinct mechanisms, from face recognition, to voice recognition, to recognizing gait and other characteristic biological motion patterns, etc. Similarly, a demanding linguistic task may engage both the core language network and the domain-general MD network (e.g., Stromswold et al., 1996; Stowe et al., 1998; Constable et al., 2004; Chen et al., 2006; Nieuwland et al., 2007; Novais-Santos et al., 2007; January et al., 2009; Ye \& Zhou, 2009; Peelle et al., 2010; Sebastian et al., 20122; Barde et al., 2012; McMillan et al., 2012, 2013; Wild et al., 2012; Brownsett et al., 2013; de Bruin et al., 2014; Geranmayeh et al., 2014a,b; De Baene et al., 2015; Gauvin et al., 2016; Henderson et al., 2016; Hsu et al., 2017); and recalling a past experience may engage both the DMN and the language network, if the experience contains verbal content. However, knowing that two brain regions that both contribute to the same complex task are functionally distinct (e.g., respond in different ways during other manipulations) is critical to understanding the precise nature of their contribution to the task in question. For example, even if the language network and DMN both support some aspects of conceptual processing (e.g., Binder et al., 2009), they plausibly differ in their respective contributions and should be probed separately when evaluating future hypotheses about semantic processing. How exactly inter-region or inter-network interactions are implemented in the brain is a topic of active research (e.g., Roelfsema et al., 1997; Canolty et al., 2010; Zalesky et al., 2014; Antzoulatos et al., 2016; Voloh \& Womelsdorf, 2016; Quax et al., 2017; Tang et al., 2017; Lundqvist et al., 2018; for reviews, see: Singer \& Gray, 1995; Tononi et al., 1998; Bressler \& Kelso, 2001; Varela et al., 2001; Fries, 2009; Canolty \& Knight, 2010; Siegel et al., 2012; Turk-Browne, 2013;

Saalmann, 2014; Bastos et al., 2015; Luczak et al., 2015; Bell \& Shine, 2016; Nakajima \& Halassa, 2017).

Second, although functional dissociations between regions or networks may imply distinct computations (e.g., Blank \& Fedorenko, 2017), the latter does not necessarily follow. In fact, neural circuits across the cortex share many core properties (e.g., Douglas et al. 1989; Douglas \& Martin 2004; Harris \& Shepherd 2015), suggesting that the basic computations may be the same or at least similar across different cortical areas even if they store or process different kinds of domain-specific representations. In addition to the kinds of analyses used here and discussed above, multivariate analyses, especially ones that relate neural activity patterns to cognitive models (e.g., Kriegeskorte et al., 2008) may help inform the representational content of different networks.

\section{Acknowledgments}

This work was supported by NIH awards R00-HD-057522 and R01- DC-016607 to E.F., and by a grant from the Simons Foundation to the Simons Center for the Social Brain at MIT. K.M. was supported by a National Defense Science and Engineering Graduate (NDSEG) Fellowship. We would like to acknowledge the Athinoula A. Martinos Imaging Center at McGovern Institute for Brain Research at MIT, and the support team (Steve Shannon, Atsushi Takahashi, and Sheeba Arnold).

\section{References}

Alexander-Bloch A, Giedd JN, \& Bullmore E (2013). Imaging structural co-variance between human brain regions. Nature Reviews Neuroscience, 14, 322-336. [PubMed: 23531697] 
Amit E, Hoeflin C, Hamzah N, \& Fedorenko E (2017). An asymmetrical relationship between verbal and visual thinking: Converging evidence from behavior and fMRI. Neuroimage, 152, 619-627. [PubMed: 28323162]

Andrews-Hanna JR, Reidler JS, Sepulcre J, Poulin R, \& Buckner RL (2010). Functional-Anatomic Fractionation of the Brain's Default Network. Neuron, 65, 550-562. [PubMed: 20188659]

Antzoulatos EG, \& Miller EK (2016). Synchronous beta rhythms of frontoparietal networks support only behaviorally relevant representations. Elife, 5, e17822. [PubMed: 27841747]

Assem M, Blank IA, Mineroff Z, Ademoglu A, \& Fedorenko E (in revision). Multiple Demand (MD) system's activity predicts individual differences in working memory and fluid intelligence. https:// www.biorxiv.org/content/early/2017/06/12/110270

Barde LH, Yeatman JD, Lee ES, Glover G, \& Feldman HM (2012). Differences in neural activation between preterm and full term born adolescents on a sentence comprehension task: implications for educational accommodations. Developmental Cognitive Neuroscience, 2, S114-S128. [PubMed: 22682901]

Bates D, Maechler M, Bolker B, \& Walker S (2015). Fitting linear mixed-effects models using lme4. Journal of Statistical Software, 67 Linear Mixed-Effects Models using 'Eigen' and S4.

Bates E, Wilson SM, Saygin AP, Dick F, Sereno MI, Knight RT, \& Dronkers NF (2003). Voxel-based lesion-symptom mapping. Nature Neuroscience, 6, 448-450. [PubMed: 12704393]

Bastos AM, Vezoli J, \& Fries P (2015). Communication through coherence with inter-areal delays. Current Opinion in Neurobiology, 31, 173-180. [PubMed: 25460074]

Bell PT, \& Shine JM (2016). Subcortical contributions to large-scale network communication. Neuroscience \& Biobehavioral Reviews, 71, 313-322. [PubMed: 27590830]

Benjamini Y, \& Yekutieli D (2001). The control of the false discovery rate in multiple testing under dependency. The Annals of Statistics, 29, 1165-1188.

Bernard JA, Seidler RD, Hassevoort KM, Benson BL, Welsh RC, Wiggins JL, Jaeggi SM, Buschkuehl M, Monk CS, Jonides J, \& Peltier SJ (2012). Resting state cortico-cerebellar functional connectivity networks: a comparison of anatomical and self-organizing map approaches. Frontiers in Neuroanatomy, 6, 31. [PubMed: 22907994]

Binder JR, Desai RH, Graves WW, \& Conant LL (2009). Where is the semantic system? A critical review and meta-analysis of 120 functional neuroimaging studies. Cerebral Cortex, 19, 27672796. [PubMed: 19329570]

Blank I, Balewski Z, Mahowald K, \& Fedorenko E, (2016). Syntactic processing is distributed across the language system. Neuroimage, 127, 307-323. [PubMed: 26666896]

Blank I, \& Fedorenko E (2017). Domain-general brain regions do not track linguistic input as closely as language-selective regions. Journal of Neuroscience, 37(41), 9999-10011. [PubMed: 28871034]

Blank I, Kanwisher N, \& Fedorenko E (2014). A functional dissociation between language and multiple-demand systems revealed in patterns of BOLD signal fluctuations. Journal of Neurophysiology, 112, 1105-1118. [PubMed: 24872535]

Braver TS, Reynolds JR, \& Donaldson DI (2003). Neural mechanisms of transient and sustained cognitive control during task switching. Neuron, 39, 713-726. [PubMed: 12925284]

Bressler SL, \& Kelso JS (2001). Cortical coordination dynamics and cognition. Trends in Cognitive Sciences, 5(1), 26-36. [PubMed: 11164733]

Brownsett SL, Warren JE, Geranmayeh F, Woodhead Z, Leech R, \& Wise RJ (2013). Cognitive control and its impact on recovery from aphasic stroke. Brain, 137(1), 242-254. [PubMed: 24163248]

Buckner RL, Andrews-Hanna JR, \& Schacter DL (2008). The brain's default network: Anatomy, function, and relevance to disease. Annals of the New York Academy of Sciences, 1124, 1-38. [PubMed: 18400922]

Buckner RL, \& Krienen FM (2013). The evolution of distributed association networks in the human brain. Trends in Cognitive Sciences, 17, 648-665. [PubMed: 24210963]

Cabeza R, \& Nyberg L (2000). Imaging cognition II: An empirical review of 275 PET and fMRI studies. Journal of Cognitive Neuroscience, 12, 1-47.

Canolty RT, Ganguly K, Kennerley SW, Cadieu CF, Koepsell K, Wallis JD, \& Carmena JM (2010). Oscillatory phase coupling coordinates anatomically dispersed functional cell assemblies. Proceedings of the National Academy of Sciences, 107(40), 17356-17361. 
Canolty RT, \& Knight RT (2010). The functional role of cross-frequency coupling. Trends in Cognitive Sciences, 14(11), 506-515. [PubMed: 20932795]

Carruthers P (2002). The cognitive functions of language. Behavioral and Brain Sciences, 25, 657674. [PubMed: 14598623]

Chai LR, Mattar MG, Blank IA, Fedorenko E, \& Bassett DS (2016). Functional network dynamics of the language system. Cerebral Cortex, 26(11), 4148-4159. [PubMed: 27550868]

Chen CH, Gutierrez ED, Thompson W, Panizzon MS, Jernigan TL, Eyler LT, Fennema-Notestine C, Jak AJ, Neale MC, Franz CE, Lyons MJ, Grant MD, Fischl B, Seidman LJ, Tsuang MT, Kremen WS, \& Dale AM (2012). Hierarchical genetic organization of human cortical surface area. Science, 335, 1634-1636. [PubMed: 22461613]

Chen E, West WC, Waters G, \& Caplan D (2006). Determinants of BOLD signal correlates of processing object-extracted relative clauses. Cortex, 42(4), 591-604. [PubMed: 16881269]

Cole MW, \& Schneider W (2007). The cognitive control network: Integrated cortical regions with dissociable functions. Neuroimage, 37, 343-360. [PubMed: 17553704]

Constable RT, Pugh KR, Berroya E, Mencl WE, Westerveld M, Ni W, \& Shankweiler D (2004). Sentence complexity and input modality effects in sentence comprehension: an fMRI study. Neuroimage, 22(1), 11-21. [PubMed: 15109993]

Crossley NA, Mechelli A, Vertes PE, Winton-Brown TT, Patel AX, Ginestet CE, McGuire P, \& Bullmore ET (2013). Cognitive relevance of the community structure of the human brain functional coactivation network. Proceedings of the National Academy of Sciences of the United States of America, 110, 11583-11588. [PubMed: 23798414]

Damasio A, \& Van Hoesen G (1983). Neuropsychology of Human Emotion. Guilford, New York.

De Baene W, Duyck W, Brass M, \& Carreiras M (2015). Brain circuit for cognitive control is shared by task and language switching. Journal of Cognitive Neuroscience, 27(9), 1752-1765. [PubMed: 25901448]

de Bruin A, Roelofs A, Dijkstra T, \& FitzPatrick I (2014). Domain-general inhibition areas of the brain are involved in language switching: FMRI evidence from trilingual speakers. Neuroimage, 90, 348-359. [PubMed: 24384153]

de Pasquale F, Della Penna S, Snyder AZ, Marzetti L, Pizzella V, Romani GL, \& Corbetta M (2012). A cortical core for dynamic integration of functional networks in the resting human brain. Neuron, 74, 753-764. [PubMed: 22632732]

Deen B, Koldewyn K, Kanwisher N, \& Saxe R (2015). Functional organization of social perception and cognition in the superior temporal sulcus. Cerebral Cortex, 25(11), 4596-4609. [PubMed: 26048954]

Dosenbach NUF, Fair DA, Miezin FM, Cohen AL, Wenger KK, Dosenbach RAT, Fox MD, Snyder AZ, Vincent JL, Raichle ME, Schlaggar BL, Petersen SE (2007). Distinct brain networks for adaptive and stable task control in humans. Proceedings of the National Academy of Sciences of the United States of America, 104, 11073-11078. [PubMed: 17576922]

Dufour N, Redcay E, Young L, Mavros PL, Moran JM, Triantafyllou C, Gabrieli JD, \& Saxe R (2013). Similar brain activation during false belief tasks in a large sample of adults with and without autism. PLoS ONE, 8, e75468. [PubMed: 24073267]

Duncan J (2010). The multiple-demand (MD) system of the primate brain: Mental programs for intelligent behaviour. Trends in Cognitive Sciences, 14, 172-179. [PubMed: 20171926]

Duncan J (2013). The structure of cognition: Attentional episodes in mind and brain. Neuron, 80, 3550. [PubMed: 24094101]

Duncan J, \& Owen AM (2000). Common regions of the human frontal lobe recruited by diverse cognitive demands. Trends in Neurosciences, 23, 475-483. [PubMed: 11006464]

Dubois J, \& Adolphs R (2016). Building a science of individual differences from fMRI. Trends in Cognitive Sciences, 20(6), 425-443. [PubMed: 27138646]

Fedorenko E (2014). The role of domain-general cognitive control in language comprehension. Frontiers in Psychology, 5, 335. [PubMed: 24803909]

Fedorenko E, Behr MK, \& Kanwisher N (2011). Functional specificity for high-level linguistic processing in the human brain. Proceedings of the National Academy of Sciences of the United States of America, 108, 16428-16433. [PubMed: 21885736] 
Fedorenko E, Duncan J, \& Kanwisher N (2012). Language-selective and domain-general regions lie side by side within Broca's area. Current Biology, 22, 2059-2062. [PubMed: 23063434]

Fedorenko E, Duncan J, \& Kanwisher N (2013). Broad domain generality in focal regions of frontal and parietal cortex. Proceedings of the National Academy of Sciences of the United States of America 110, 16616-16621. [PubMed: 24062451]

Fedorenko E, Hsieh PJ, Nieto-Castanon A, Whitfield-Gabrieli S, \& Kanwisher N (2010). New method for fMRI investigations of language: Defining ROIs functionally in individual subjects. Journal of Neurophysiology, 104, 1177-1194. [PubMed: 20410363]

Fedorenko E, \& Thompson-Schill SL (2014). Reworking the language network. Trends in Cognitive Sciences, 18(3), 120-126. [PubMed: 24440115]

Fedorenko E, \& Varley R (2016). Language and thought are not the same thing: Evidence from neuroimaging and neurological patients. Annals of the New York Academy of Sciences, 1369, 132-153. [PubMed: 27096882]

Fox MD, Snyder AZ, Vincent JL, Corbetta M, Van Essen DC, \& Raichle ME (2005). The human brain is intrinsically organized into dynamic, anticorrelated functional networks. Proceedings of the National Academy of Sciences of the United States of America, 102, 9673-9678. [PubMed: 15976020]

Fransson P (2005). Spontaneous low-frequency BOLD signal fluctuations: an fMRI investigation of the resting-state default mode of brain function hypothesis. Human Brain Mapping, 26, 15-29. [PubMed: 15852468]

Fries P (2009). Neuronal gamma-band synchronization as a fundamental process in cortical computation. Annual Review of Neuroscience, 32, 209-224.

Gauvin HS, De Baene W, Brass M, \& Hartsuiker RJ (2016). Conflict monitoring in speech processing: An fMRI study of error detection in speech production and perception. NeuroImage, 126, 96-105. [PubMed: 26608243]

Geranmayeh F, Brownsett SL, \& Wise RJ (2014a). Task-induced brain activity in aphasic stroke patients: what is driving recovery?. Brain, 137(10), 2632-2648. [PubMed: 24974382]

Geranmayeh F, Wise RJ, Mehta A, \& Leech R (2014b). Overlapping networks engaged during spoken language production and its cognitive control. Journal of Neuroscience, 34(26), 8728-8740. [PubMed: 24966373]

Gibson E (1998). Linguistic complexity: Locality of syntactic dependencies. Cognition, 68(1), 1-76. [PubMed: 9775516]

Golland Y, Bentin S, Gelbard H, Benjamini Y, Heller R, Nir Y, Hasson U, \& Malach R (2007). Extrinsic and intrinsic systems in the posterior cortex of the human brain revealed during natural sensory stimulation. Cerebral Cortex, 17, 766-777. [PubMed: 16699080]

Gotts SJ, Jo HJ, Wallace GL, Saad ZS, Cox RW, \& Martin A (2013). Two distinct forms of functional lateralization in the human brain. Proceedings of the National Academy of Sciences of the United States of America, 110, E3435-3444. [PubMed: 23959883]

Greicius MD, Krasnow B, Reiss AL, \& Menon V (2003). Functional connectivity in the resting brain: A network analysis of the default mode hypothesis. Proceedings of the National Academy of Sciences of the United States of America, 100, 253-258. [PubMed: 12506194]

Gusnard DA, \& Raichle ME (2001). Searching for a baseline: functional imaging and the resting human brain. Nature Reviews Neuroscience, 2, 685-694. [PubMed: 11584306]

Gómez S, Jensen P, \& Arenas A (2009). Analysis of community structure in networks of correlated data. Physical Review E: Statistical, Nonlinear, Biological, and Soft Matter Physics, 80, 016114.

Hagmann P, Cammoun L, Gigandet X, Meuli R, Honey CJ, Wedeen VJ, \& Sporns O (2008). Mapping the structural core of human cerebral cortex. PLOS Biology, 6, e159. [PubMed: 18597554]

Hampshire A, Highfield RR, Parkin BL, \& Owen AM (2012). Fractionating human intelligence. Neuron, 76, 1225-1237. [PubMed: 23259956]

Hampson M, Peterson BS, Skudlarski P, Gatenby JC, \& Gore JC (2002). Detection of functional connectivity using temporal correlations in MR images. Human Brain Mapping, 15, 247-262. [PubMed: 11835612]

Hartigan J (1975). Clustering Algorithms. Wiley, New York. 
Henderson JM, Choi W, Lowder MW, \& Ferreira F (2016). Language structure in the brain: A fixationrelated fMRI study of syntactic surprisal in reading. Neuroimage, 132, 293-300. [PubMed: 26908322]

Hsu NS, Jaeggi SM, \& Novick JM (2017). A common neural hub resolves syntactic and non-syntactic conflict through cooperation with task-specific networks. Brain and Language, 166, 63-77. [PubMed: 28110105]

Hsu NS, \& Novick JM (2016). Dynamic engagement of cognitive control modulates recovery from misinterpretation during real-time language processing. Psychological Science, 27(4), 572-582. [PubMed: 26957521]

Hugdahl K, Raichle ME, Mitra A, \& Specht K (2015). On the existence of a generalized non-specific task-dependent network. Frontiers in Human Neuroscience, 9, 430. [PubMed: 26300757]

Humphreys GF, Hoffman P, Visser M, Binney RJ, \& Lambon Ralph MA (2015). Establishing task- and modality-dependent dissociations between the semantic and default mode networks. Proceedings of the National Academy of Sciences of the United States of America, 112, 7857-7862. [PubMed: 26056304]

Jackson RL, Hoffman P, Pobric G, \& Lambon Ralph MA (2016). The Semantic Network at Work and Rest: Differential Connectivity of Anterior Temporal Lobe Subregions. Journal of Neuroscience, 36, 1490-1501. [PubMed: 26843633]

January D, Trueswell JC, \& Thompson-Schill SL (2009). Co-localization of Stroop and syntactic ambiguity resolution in Broca's area: Implications for the neural basis of sentence processing. Journal of Cognitive Neuroscience, 21(12), 2434-2444. [PubMed: 19199402]

Julian JB, Fedorenko E, Webster J, \& Kanwisher N (2012). An algorithmic method for functionally defining regions of interest in the ventral visual pathway. Neuroimage, 60, 2357-2364. [PubMed: 22398396]

Kalcher K, Huf W, Boubela RN, Filzmoser P, Pezawas L, Biswal B, Kasper S, Moser E, \& Windischberger C (2012). Fully exploratory network independent component analysis of the 1000 functional connectomes database. Frontiers in Human Neuroscience, 6, 301. [PubMed: 23133413]

Konopka G, Friedrich T, Davis-Turak J, Winden K, Oldham MC, Gao F, Chen L, Wang GZ, Luo R, Preuss TM, \& Geschwind DH (2012). Human-specific transcriptional networks in the brain. Neuron, 75, 601-617. [PubMed: 22920253]

Kriegeskorte N, Simmons WK, Bellgowan PS, \& Baker CI (2009). Circular analysis in systems neuroscience: the dangers of double dipping. Nature Neuroscience, 12, 535-540. [PubMed: 19396166]

Leech R, Kamourieh S, Beckmann CF, \& Sharp DJ (2011). Fractionating the default mode network: distinct contributions of the ventral and dorsal posterior cingulate cortex to cognitive control. Journal of Neuroscience, 31, 3217-3224. [PubMed: 21368033]

Luczak A, McNaughton BL, \& Harris KD (2015). Packet-based communication in the cortex. Nature Reviews Neuroscience, 16(12), 745-755. [PubMed: 26507295]

Lundqvist M, Herman P, Warden MR, Brincat SL, \& Miller EK (2018). Gamma and beta bursts during working memory readout suggest roles in its volitional control. Nature Communications, 9(1), 394.

Mahowald K, \& Fedorenko E (2016). Reliable individual-level neural markers of high-level language processing: A necessary precursor for relating neural variability to behavioral and genetic variability. NeuroImage, 139, 74-93. [PubMed: 27261158]

Mantini D, Gerits A, Nelissen K, Durand JB, Joly O, Simone L, Sawamura H, Wardak C, Orban GA, Buckner RL, \& Vanduffel W (2011). Default mode of brain function in monkeys. Journal of Neuroscience, 31(36), 12954-12962. [PubMed: 21900574]

Mattheiss SR, Levinson H, \& Graves WW (2018). Duality of Function: Activation for Meaningless Nonwords and Semantic Codes in the Same Brain Areas. Cerebral Cortex, 28(7), 2516-2524. [PubMed: 29901789]

McKiernan KA, Kaufman JN, Kucera-Thompson J, \& Binder JR (2003). A parametric manipulation of factors affecting task-induced deactivation in functional neuroimaging. Journal of Cognitive Neuroscience, 15, 394-408. [PubMed: 12729491] 
McMillan CT, Clark R, Gunawardena D, Ryant N, \& Grossman M (2012). fMRI evidence for strategic decision-making during resolution of pronoun reference. Neuropsychologia, 50(5), 674-687. [PubMed: 22245014]

McMillan CT, Coleman D, Clark R, Liang TW, Gross RG, \& Grossman M (2013). Converging evidence for the processing costs associated with ambiguous quantifier comprehension. Frontiers in Psychology, 4, 153. [PubMed: 23565102]

Menenti L, Gierhan SM, Segaert K, \& Hagoort P (2011). Shared language: overlap and segregation of the neuronal infrastructure for speaking and listening revealed by functional MRI. Psychological Science, 22(9), 1173-1182 [PubMed: 21841148]

Mesulam M-M, Thompson CK, Weintraub S, \& Rogalski EJ (2015). The Wernicke conundrum and the anatomy of language comprehension in primary progressive aphasia. Brain, 138, 2423-2437. [PubMed: 26112340]

Mirman D, Chen Q, Zhang Y, Wang Z, Faseyitan OK, Coslett HB, \& Schwartz MF (2015). Neural organization of spoken language revealed by lesion-symptom mapping. Nature Communications, 6, 7762 .

Mitchell DJ, Bell AH, Buckley MJ, Mitchell AS, Sallet J, \& Duncan J (2016). A putative multipledemand system in the macaque brain. Journal of Neuroscience, 36(33), 8574-8585. [PubMed: 27535906]

Monti MM, Parsons LM, \& Osherson DN (2012). Thought beyond language: Neural dissociation of algebra and natural language. Psychological Science, 23, 914-922. [PubMed: 22760883]

Morin A, \& Michaud J (2007). Self-awareness and the left inferior frontal gyrus: inner speech use during self-related processing. Brain Research Bulletin, 74, 387-396. [PubMed: 17920447]

Nakajima M, \& Halassa MM (2017). Thalamic control of functional cortical connectivity. Current Opinion in Neurobiology, 44, 127-131. [PubMed: 28486176]

Newman ME, \& Girvan M (2004). Finding and evaluating community structure in networks. Physical Review E: Statistical, Nonlinear, Biological, and Soft Matter Physics, 69, 026113.

Nieto-Castañón A, \& Fedorenko E (2012). Subject-specific functional localizers increase sensitivity and functional resolution of multi-subject analyses. NeuroImage, 63, 1646-1669. [PubMed: 22784644]

Nieuwland MS, Petersson KM, \& Van Berkum JJ (2007). On sense and reference: Examining the functional neuroanatomy of referential processing. NeuroImage, 37(3), 993-1004. [PubMed: 17611124]

Novais-Santos S, Gee J, Shah M, Troiani V, Work M, \& Grossman M (2007). Resolving sentence ambiguity with planning and working memory resources: Evidence from fMRI. NeuroImage, 37(1), 361-378. [PubMed: 17574445]

Novick JM, Trueswell JC, \& Thompson-Schill SL (2010). Broca's area and language processing: Evidence for the cognitive control connection. Language and Linguistics Compass, 4, 906-924.

Nozari N, \& Novick JM (2017). Monitoring and control in language production. Current Directions in Psychological Science, 26(5), 403-410.

Ojemann SG, Berger MS, Lettich E, \& Ojemann GA (2003). Localization of language function in children: results of electrical stimulation mapping. Journal of Neurosurgery, 98, 465-470. [PubMed: 12650415]

Park DC, Polk TA, Hebrank AC, \& Jenkins LJ (2010). Age differences in default mode activity on easy and difficult spatial judgment tasks. Frontiers in Human Neuroscience, 3, 75. [PubMed: 20126437]

Paunov A, Blank IA, \& Fedorenko E (in revision). Functionally distinct language and Theory of Mind networks synchronize at rest and during language comprehension. https://psyarxiv.com/eb7wp/

Peelle JE, Troiani V, Wingfield A, \& Grossman M (2009). Neural processing during older adults' comprehension of spoken sentences: age differences in resource allocation and connectivity. Cerebral Cortex, 20(4), 773-782. [PubMed: 19666829]

Philippi CL, Tranel D, Duff M, \& Rudrauf D (2015). Damage to the default mode network disrupts autobiographical memory retrieval. Social Cognitive and Affective Neuroscience, 10, 318-326. [PubMed: 24795444] 
Pléh C (2002). Speech as an opportunistic vehicle of thinking. Behavioral and Brain Sciences, 25, 695-696.

Poldrack RA (2006). Can cognitive processes be inferred from neuroimaging data? Trends in Cognitive Sciences, 10, 59-63. [PubMed: 16406760]

Poldrack RA (2011). Inferring mental states from neuroimaging data: from reverse inference to largescale decoding. Neuron, 72, 692-697. [PubMed: 22153367]

Power JD, Cohen AL, Nelson SM, Wig GS, Barnes KA, Church JA, Vogel AC, Laumann TO, Miezin FM, Schlaggar BL, \& Petersen SE (2011). Functional network organization of the human brain. Neuron, 72, 665-678. [PubMed: 22099467]

Pritchett B, Hoeflin C, Koldewyn K, Dechter E, \& Fedorenko E (in press). High-level language processing regions are not engaged in action observation or imitation. Journal of Neurophysiology.

Quax S, Jensen O, \& Tiesinga P (2017). Top-down control of cortical gamma-band communication via pulvinar induced phase shifts in the alpha rhythm. PLoS Computational Biology, 13(5), e1005519. [PubMed: 28472057]

Raichle ME, MacLeod AM, Snyder AZ, Powers WJ, Gusnard DA, \& Shulman GL (2001). A default mode of brain function. Proceedings of the National Academy of Sciences of the United States of America, 98, 676-682. [PubMed: 11209064]

Raznahan A, Lerch JP, Lee N, Greenstein D, Wallace GL, Stockman M, Clasen L, Shaw PW, \& Giedd JN (2011). Patterns of coordinated anatomical change in human cortical development: a longitudinal neuroimaging study of maturational coupling. Neuron, 72, 873-884. [PubMed: 22153381]

Roelfsema PR, Engel AK, Konig P, \& Singer W (1997). Visuomotor integration is associated with zero time-lag synchronization among cortical areas. Nature, 385(6612), 157-161. [PubMed: 8990118]

Saalmann YB (2014). Intralaminar and medial thalamic influence on cortical synchrony, information transmission and cognition. Frontiers in Systems Neuroscience, 8, 83. [PubMed: 24847225]

Saxe R, Moran JM, Scholz J, \& Gabrieli J (2006). Overlapping and non-overlapping brain regions for theory of mind and self reflection in individual subjects. Social Cognitive and Affective Neuroscience, 1, 229-234. [PubMed: 18985110]

Schrauf R (2002). Bilingual inner speech as the medium of cross-modular retrieval in autobiographical memory. Behavioral and Brain Sciences, 25, 698-699.

Scott TL, Gallee J, \& Fedorenko E (2016). A new fun and robust version of an fMRI localizer for the frontotemporal language system. Cognitive Neuroscience, 8(3), 167-176. [PubMed: 27386919]

Sebastian R, Laird AR, \& Kiran S (2011). Meta-analysis of the neural representation of first language and second language. Applied Psycholinguistics, 32(4), 799-819

Seeley WW, Crawford RK, Zhou J, Miller BL, \& Greicius MD (2009). Neurodegenerative diseases target large-scale human brain networks. Neuron, 62, 42-52. [PubMed: 19376066]

Seeley WW, Menon V, Schatzberg AF, Keller J, Glover GH, Kenna H, Reiss AL, \& Greicius MD (2007). Dissociable intrinsic connectivity networks for salience processing and executive control. Journal of Neuroscience, 27, 2349-2356. [PubMed: 17329432]

Seghier ML, \& Price CJ (2018). Interpreting and utilising intersubject variability in brain function. Trends in Cognitive Sciences, 22(6), 517-530. [PubMed: 29609894]

Siegel M, Donner TH, \& Engel AK (2012). Spectral fingerprints of large-scale neuronal interactions. Nature Reviews Neuroscience, 13(2), 121-134. [PubMed: 22233726]

Silver NC, \& Dunlap WP (1987). Averaging correlation coefficients: Should Fisher's z transformation be used? Journal of Applied Psychology, 72, 146-148.

Singer W, \& Gray CM (1995). Visual feature integration and the temporal correlation hypothesis. Annual Review of Neuroscience, 18(1), 555-586.

Sokolov A (1972). Inner Speech and Thought. Translated by Onischenko George T.. Springer, New York.

Spreng RN, Mar RA, \& Kim AS (2009). The common neural basis of autobiographical memory, prospection, navigation, theory of mind, and the default mode: a quantitative meta-analysis. Journal of Cognitive Neuroscience, 21, 489-510. [PubMed: 18510452] 
Stiers P, Mennes M, \& Sunaert S (2010). Distributed task coding throughout the multiple demand network of the human frontal-insular cortex. Neuroimage, 52, 252-262. [PubMed: 20362676]

Stowe LA, Broere CA, Paans AM, Wijers AA, Mulder G, Vaalburg W, \& Zwarts F (1998). Localizing components of a complex task: sentence processing and working memory. Neuroreport, $9(13)$, 2995-2999. [PubMed: 9804304]

Stromswold K, Caplan D, Alpert N, \& Rauch S (1996). Localization of syntactic comprehension by positron emission tomography. Brain and Language, 52(3), 452-473 [PubMed: 8653390]

Tang E, Giusti C, Baum GL, Gu S, Pollock E, Kahn AE, Roalf DR, Moore TM, Ruparel K, Gur RC, \& Gur RE (2017). Developmental increases in white matter network controllability support a growing diversity of brain dynamics. Nature Communications, 8(1), 1252.

Tononi G, Edelman GM, \& Sporns O (1998). Complexity and coherency: integrating information in the brain. Trends in Cognitive Sciences, 2(12), 474-484. [PubMed: 21227298]

Turk-Browne NB (2013). Functional interactions as big data in the human brain. Science, 342(6158), 580-584. [PubMed: 24179218]

Toro R, Fox PT, \& Paus T (2008). Functional coactivation map of the human brain. Cerebral Cortex, 18, 2553-2559. [PubMed: 18296434]

Turken AU, \& Dronkers NF (2011). The neural architecture of the language comprehension network: converging evidence from lesion and connectivity analyses. Frontiers in Systems Neuroscience, 5, 1. [PubMed: 21347218]

Tzourio-Mazoyer N, Landeau B, Papathanassiou D, Crivello F, Etard O, Delcroix N, Mazoyer B, Joliot M (2002). Automated anatomical labeling of activations in SPM using a macroscopic anatomical parcellation of the MNI MRI single-subject brain. NeuroImage, 15, 273-289. [PubMed: 11771995]

Uddin LQ, Kelly AM, Biswal BB, Castellanos FX, Milham MP (2009). Functional connectivity of default mode network components: correlation, anticorrelation, and causality. Human Brain Mapping, 30, 625-637. [PubMed: 18219617]

van den Heuvel MP, \& Sporns O (2011). Rich-club organization of the human connectome. Journal of Neuroscience, 31, 15775-15786. [PubMed: 22049421]

Varela F, Lachaux JP, Rodriguez E, Martinerie J (2001). The brainweb: phase synchronization and large-scale integration. Nature Reviews Neuroscience, 2(4), 229-239. [PubMed: 11283746]

Voloh B, \& Womelsdorf T (2016). A role of phase-resetting in coordinating large scale neural networks during attention and goal-directed behavior. Frontiers in Systems Neuroscience, 10, 18. [PubMed: 27013986]

Vygotsky LS, (1962/2012). Thought and Language. MIT Press, Cambridge, MA.

Vértes PE, Alexander-Bloch AF, Gogtay N, Giedd JN, Rapoport JL, \& Bullmore ET (2012). Simple models of human brain functional networks. Proceedings of the National Academy of Sciences of the United States of America, 109, 5868-5873. [PubMed: 22467830]

Wild CJ, Yusuf A, Wilson DE, Peelle JE, Davis MH, \& Johnsrude IS (2012). Effortful listening: the processing of degraded speech depends critically on attention. Journal of Neuroscience, 32(40), 14010-14021. [PubMed: 23035108]

Wirth M, Jann K, Dierks T, Federspiel A, Wiest R, \& Horn H (2011). Semantic memory involvement in the default mode network: a functional neuroimaging study using independent component analysis. Neuroimage, 54, 3057-3066. [PubMed: 20965253]

Wu K, Taki Y, Sato K, Sassa Y, Inoue K, Goto R, Okada K, Kawashima R, He Y, Evans AC, \& Fukuda $\mathrm{H}$ (2011). The overlapping community structure of structural brain network in young healthy individuals. PLoS ONE, 6, e19608. [PubMed: 21573111]

Ye Z, \& Zhou X (2009). Conflict control during sentence comprehension: fMRI evidence. Neuroimage, 48(1), 280-290 [PubMed: 19540923]

Yeo BT, Krienen FM, Sepulcre J, Sabuncu MR, Lashkari D, Hollinshead M, Roffman JL, Smoller JW, Zollei L, Polimeni JR, Fischl B, Liu H, \& Buckner RL (2011). The organization of the human cerebral cortex estimated by intrinsic functional connectivity. Journal of Neurophysiology, 106, 1125-1165. [PubMed: 21653723] 
Yue Q, Zhang L, Xu G, Shu H, \& Li P (2013). Task-modulated activation and functional connectivity of the temporal and frontal areas during speech comprehension. Neuroscience, 237, 87-95. [PubMed: 23357111]

Zalesky A, Fornito A, Cocchi L, Gollo LL, \& Breakspear M (2014). Time-resolved resting-state brain networks. Proceedings of the National Academy of Sciences, 111(28), 10341-10346.

Zilles K, Bacha-Trams M, Palomero-Gallagher N, Amunts K, \& Friederici AD (2015). Common molecular basis of the sentence comprehension network revealed by neurotransmitter receptor fingerprints. Cortex, 63, 79-89. [PubMed: 25243991]

Zivin G (1979). The Development of Self-Regulation through Private Speech. Wiley, New York. 


\section{Highlights:}

- Is the language network dissociable from multiple-demand and default mode networks?

- $\quad$ Novel test: do individual differences in effect size (ES) correlate across regions?

- Individual differences co-vary within networks much more than between networks

- Data-driven support for a triple language/multiple-demand/default mode dissociation

- Individual differences in regional ES respect the brain's functional organization 

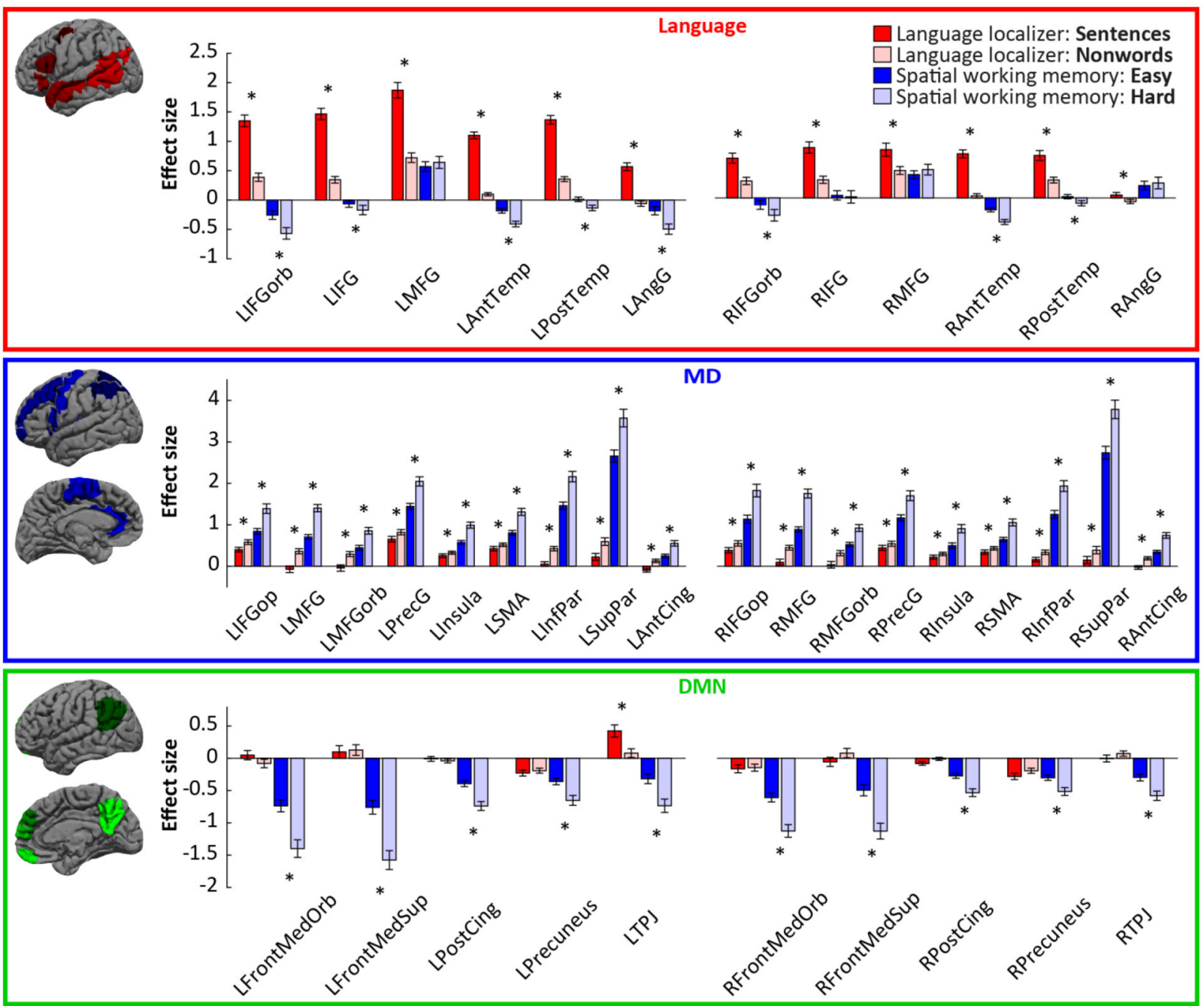

Figure 1.

Responses to the conditions of the localizer tasks in each functional ROI. Broad masks used to constrain the selection of subject-specific fROIs are shown on the left (the size of fROIs is $10 \%$ of the size of the masks). Language fROIs (top) are defined by the sentences > nonwords contrast; MD fROIs (middle) are defined by the hard > easy spatial working memory contrast; and DMN fROIs (bottom) are defined by the easy $>$ hard spatial working memory contrast. Responses to the conditions used for defining the fROIs are estimated using across-runs cross-validation, to ensure independence. Left: left-hemispheric fROIs (L prefix). Right: right-hemispheric fROIs (R prefix). Significant effects (after an FDRcorrection for multiple comparisons within each network) are marked with an asterisk. IFG: inferior frontal gyrus; IFGorb: IFG pars orbitalis; MFG: middle frontal gyrus; AntTemp: anterior temporal cortex; PostTemp: posterior temporal cortex; AngG: angular gyrus; IFGop: IFG pars opercularis; MFGorb: MFG, orbital part; PrecG: precentral gyrus; SMA: supplementary motor area; InfPar: inferior parietal cortex; SupPar: superior parietal cortex; AntCing: anterior cingulate cortex; FrontMedOrb: medial frontal cortex, orbital part; FrontMedSup: medial frontal cortex, superior part; PostCing: posterior cingulate cortex; TPJ: temporoparietal junction. 


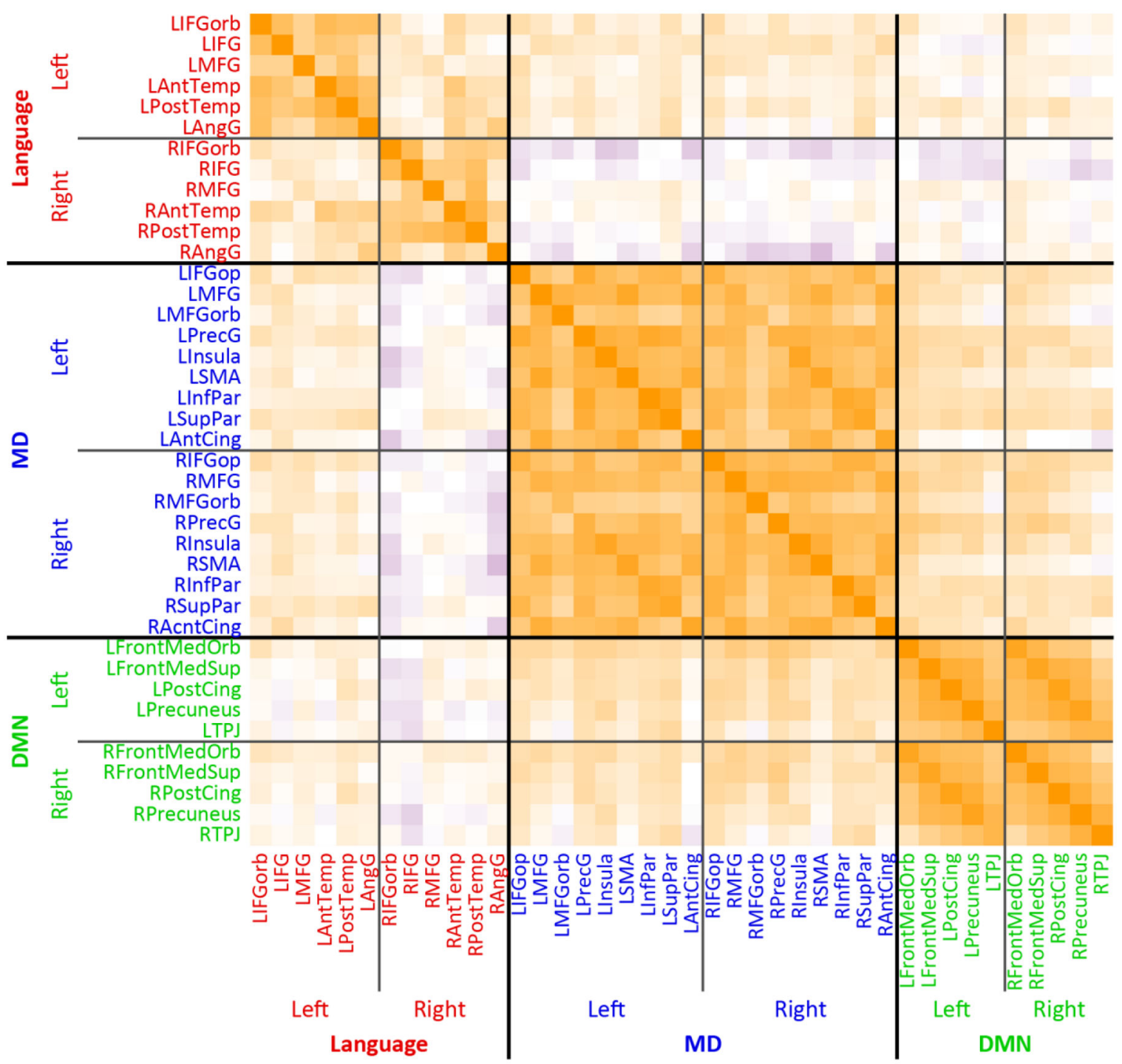

Pearson's correlation coefficient in effect size across participants

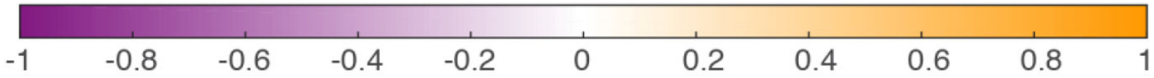

Figure 2.

Pearson correlations, across 60 participants, for effect sizes of localizer contrasts across pairs of fROIs. For each fROI, the effect size is for the contrast used to define that fROI (but estimated in independent data): for the 12 language regions (labeled in red font), the sentences > nonwords contrast from the language localizer task was used; for the MD regions (blue font), the hard > easy spatial WM contrast was used; and for the DMN regions (green font), the easy $>$ hard spatial WM contrast was used. 


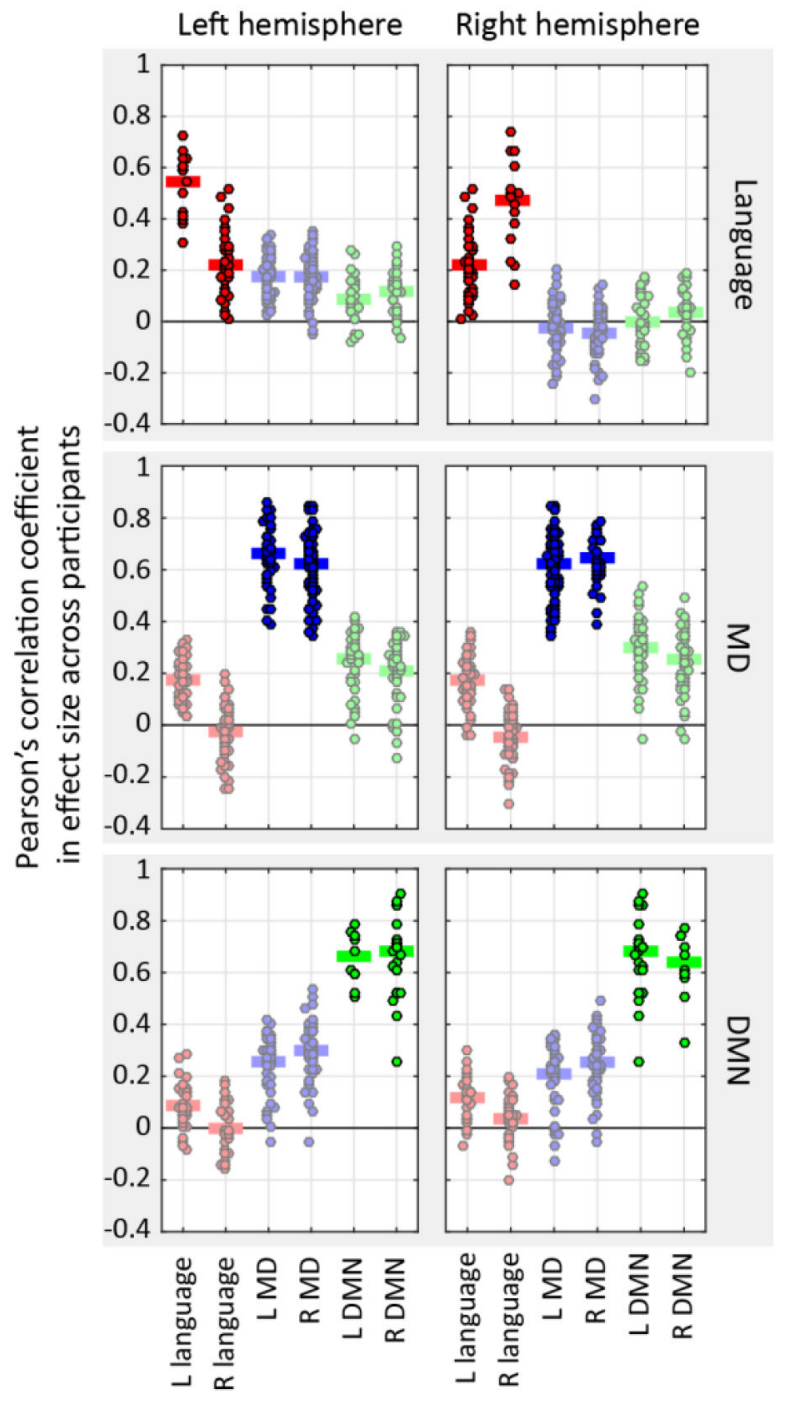

Figure 3.

Correlations in effect sizes across participants, computed either within networks (strong colors) or between networks (faint colors). Each point is a pairwise correlation between the effect sizes of two fROIs, one from the network/hemisphere denoted on the $x$-axis, and one from the network/hemisphere denoted by the subplot titles (top: language; middle: MD; bottom: DMN; left: left hemisphere; right: right hemisphere). Horizontal lines show averages across these pairwise correlations. 


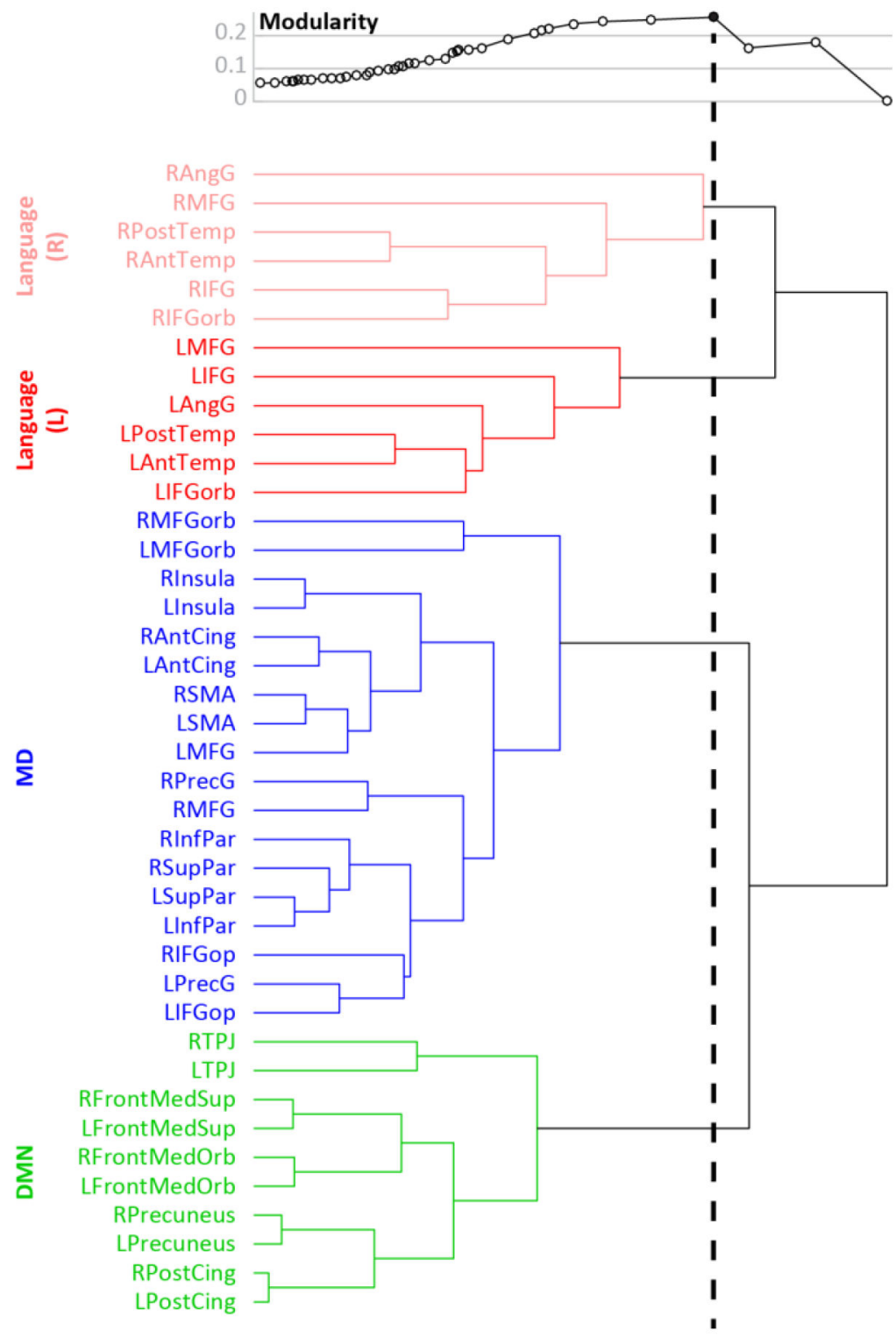

Figure 4.

Hierarchical clustering results. In the binary tree shown, branch length (i.e., horizontal lines) corresponds to the similarity between fROIs (or sets of fROIs). Above the tree, modularity is plotted for all fROI partitions licensed by the tree. Each point on the modularity plot corresponds to a partition generated by drawing an imaginary vertical line from that point through the tree and clustering together only those fROIs that are merged to the left of this line (fROIs that are merged to the right of the line remain in separate clusters). A sample vertical line is drawn for the maximal modularity. 
Table 1.

Mean inter-regional correlations in contrast-specific effect sizes across participants ${ }^{a}$

\begin{tabular}{lcc}
\hline & \multicolumn{2}{c}{$r$} \\
Localizer contrast & LH & RH \\
\hline Reading: Sentences > Nonwords & & \\
$\quad$ Within language & 0.54 & 0.47 \\
Language vs. MD & 0.10 & 0.16 \\
Language vs. DMN & 0.30 & 0.09 \\
Spatial WM: Hard > Easy & & \\
Within MD & 0.66 & 0.65 \\
MD vs. language & 0.10 & 0.13 \\
MD vs. DMN & -0.26 & -0.25 \\
Spatial WM: Easy > Hard & & \\
Within DMN & 0.66 & 0.64 \\
DMN vs. language & 0.38 & 0.25 \\
DMN vs. MD & -0.26 & -0.25 \\
\hline
\end{tabular}

${ }^{a}$ All within-network correlations are stronger than their respective across-network correlations at $p<10^{-7}$ 